\title{
Anabases
}

ANABASES Traditions et réceptions de l'Antiquité

$14 \mid 2011$

Varia

\section{Aude Doody, Pliny's Encyclopedia: The Reception of the Natural History}

\section{Germaine Aujac}

\section{OpenEdition}

\section{Journals}

Édition électronique

URL : http://journals.openedition.org/anabases/2394

DOI : 10.4000/anabases.2394

ISSN : 2256-9421

\section{Éditeur}

E.R.A.S.M.E.

\section{Édition imprimée}

Date de publication : 1 octobre 2011

Pagination : 258-260

ISSN : 1774-4296

\section{Référence électronique}

Germaine Aujac, "Aude doody, Pliny's Encyclopedia : The Reception of the Natural History ", Anabases [En ligne], 14 | 2011, mis en ligne le 01 octobre 2011, consulté le 22 septembre 2020. URL : http:// journals.openedition.org/anabases/2394 ; DOI : https://doi.org/10.4000/anabases.2394

Ce document a été généré automatiquement le 22 septembre 2020.

(c) Anabases 


\title{
Aude Doody, Pliny's Encyclopedia: The Reception of the Natural History
}

\author{
Germaine Aujac
}

\section{RÉFÉRENCE}

Aude DOODY, Pliny's Encyclopedia: The Reception of the Natural History, Cambridge-New

York, Cambridge University Press, 2010, 194 p.

95 dollars / ISBN 978-0-521-49103-7.

1 L'étude de la réception de l'Histoire naturelle de Pline permet à Aude Doody d'ouvrir de larges perspectives sur la survie, au cours des siècles, de cette vaste encyclopédie que constituent les trente-sept livres d'un auteur plus connu pour sa fin tragique que lu dans le seul ouvrage de lui qui ait été conservé.

La première question posée par Aude Doody : l'Histoire naturelle est-elle véritablement une encyclopédie, lui fournit l'occasion de définir ce terme qui est de toute évidence de création tardive. L'Histoire naturelle n'en est une, pense-t-elle, que parce qu'elle est lue comme telle. Dans l'introduction (p.1-10), l'A. dit son intention d'isoler quelques moments significatifs de la réception de Pline : ils sont symbolisés par Francis Bacon, puis par Diderot, mais aussi par les divers traitements qu'ont fait subir les éditeurs au Sommaire qui occupe le Livre I de l'Histoire naturelle (serait-ce une table des matières ?), ainsi que par la floraison d'extraits relatifs à certaines spécialités, l'art ou la médecine, pour ne prendre que ces exemples.

3 Le chapitre 1, intitulé "Science and encyclopedism: the originality of the Natural History » (p. 11-39), souligne l'ambiguïté du terme encyclopédie, qui n'apparaît qu'en 1559 chez Paul Scaliger: désigne-t-il un savoir universel, ou la réunion de tous les matériaux disponibles dans un domaine spécial ? L'érudition de Pline, qui se réduisait souvent à un simple catalogue de détails, sans idée générale (p. 26-28), eut un grand succès au Moyen Âge. Chez lui, pourtant, la Nature est partout présente, dans les petites choses comme dans les grandes. Les listes de noms sont organisées suivant un 
principe de hiérarchie : le monde est ordonné. Pline cherche à procurer du plaisir au lecteur par la contemplation de la Nature (p. 30). Après la vogue qu'a connue l'Histoire naturelle au Moyen Âge, et des imitations plus ou moins réussies par des compilateurs comme Isidore de Séville (vers 560-636) ou Vincent de Beauvais (vers 1190-1264), vint une réaction initiée par Francis Bacon (1561-1626). Ce savant original préférait à l'autorité des Anciens le recours à l'expérimentation, comme le montre son Novum Organum (livre II). Les trois facultés essentielles pour un scientifique sont, à son avis, la Mémoire, la Raison, l'Imagination. Il reproche donc à Pline de cataloguer tout le savoir, sans le trier; il l'accuse d'accorder trop d'importance, dans son récit, aux mirabilia. La défaveur qui frappa Pline au $\mathrm{XVI}^{\mathrm{e}}$ siècle vint de ce que l'on mettait alors l'accent sur l'observation personnelle, premier pas vers la Révolution Scientifique.

"Diderot's Pliny and the politics of the encyclopedia » font l'objet du chapitre 2 (p. 40-91). L'Histoire naturelle, contrairement aux ouvrages de Caton, Varron ou Celse avec lesquels on la compare souvent, n'est pas consacrée à une science particulière. C'est dans ce sens que l'on peut y voir un exemple d'enkuklios paideia qui, pour les Anciens, était une éducation générale, préliminaire à des études plus spécialisées. Elle serait, pour Trevor Murphy (Pliny the Elder's Natural History. The Empire in the Encyclopedia, Oxford Univ. Pr., 2004), un document politique, produit culturel de l'empire romain, analogue à ce que fut, pour l'empire britannique, l'Encyclopedia Britannica, née à Édimbourg en 1771.

5 C'est en 1750 que parut le Prospectus annonçant la publication prochaine, par les soins de Denis Diderot et de Jean Le Rond D'Alembert, de l'Encyclopédie ou Dictionnaire raisonné des sciences, des arts et des métiers, qui devait jouer un rôle primordial dans le changement des esprits conduisant à la Révolution Française. L'ouvrage se présentait comme une simple traduction de la Cyclopedia de l'anglais Chambers (mort en 1740), mais ses éditeurs lui donnèrent très vite une bien plus large extension; à la fois technique et philosophique, l'Encyclopédie devait favoriser la démocratisation du savoir et aborder toutes sortes de sujets, classés par ordre alphabétique, et traités par un grand nombre de collaborateurs. Publiée quelque dix-sept ans après le Prospectus, elle adressait des critiques voilées à la religion, et au pouvoir en place, d'où la référence à Pline, obligé lui aussi de dissimuler ses opinions pour ne pas mettre en danger sa tranquillité (p. 78). Dans l'article consacré à l'Histoire Naturelle, Diderot fait de Pline « un des plus grands philosophes de l'Antiquité ». À l'article «Vérone », lieu probable de sa naissance, le chevalier de Jaucourt évoque Pline, érudit accompli et martyr de la science. Mais l'opinion de Diderot, qui défendait la supériorité de l'art ancien, tel qu'il était présenté par Pline, fut contestée vigoureusement par le sculpteur Falconet (1716-1791), auteur d'une célèbre statue équestre de Pierre le Grand : des lettres furent échangées, en une sorte de querelle des Anciens et des Modernes qui contribua grandement à affaiblir le prestige de Pline et à faire douter de sa fiabilité.

6 Le chapitre 3, "Finding facts: the Summarium in the early printed editions" (p. 90-131), passe en revue les diverses présentations du Sommaire; d'après Pline, ce Sommaire devait permettre d'aller directement à ce que l'on cherchait, et de savoir à chaque fois quelles sources, latines ou autres, avaient été utilisées. Mais le Sommaire, qui remplit le livre I dans les manuscrits, pourrait-il être distribué au début de chaque livre? Des réponses diverses ont été apportées par les éditeurs successifs, chaque procédé présentant des avantages et des inconvénients qu'analyse l'A. avec grande pertinence. 
7 Le chapitre 4, "Specialist readings: art and medicine from the Natural History" (p.132-172), montre comment l'ouvrage de Pline fut utilisé pour constituer des répertoires de spécialités. L'A. choisit deux thèmes, l'histoire de l'art et la médecine. Dès le $\mathrm{IV}^{\mathrm{e}}$ siècle, une Medicina Plinii fut composée, en trois livres; enrichie au $\mathrm{VI}^{\mathrm{e}}$ siècle, elle fut publiée par Alban Thorer, De re medica, en 1528 et connut un grand succès. Quant aux chapitres de Pline sur l'histoire de l'art, traduits par Katherine Jex-Blade, ils furent édités par Eugénie Sellers ; publiés en 1896, ils furent réimprimés deux fois au $\mathrm{XX}^{\mathrm{e}}$ siècle et sont encore très souvent utilisés.

8 L'A. insiste en concluant sur l'importance exceptionnelle de l'œuvre de Pline, riche d'une collection incomparable d'informations variées, qui ont nourri déjà tant de générations successives. Le tableau qu'elle nous offre, des diverses approches du texte, illustre magnifiquement son projet de faire une étude d'histoire intellectuelle. L'Histoire Naturelle mérite d'être lue, à ses yeux, non pas tant pour l'information qu'on y trouve, ou encore pour sa vision politique, que pour son statut intellectuel dans la Rome du $\mathrm{I}^{\mathrm{er}}$ siècle.

\section{AUTEURS}

\section{GERMAINE AUJAC}

Université de Toulouse (UTM)

aujac.germaine@wanadoo.fr 